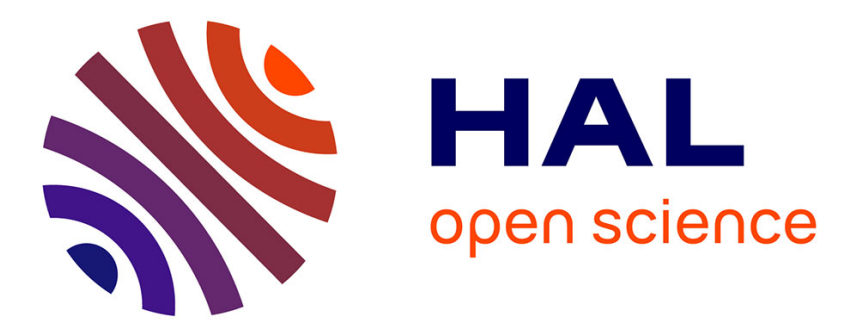

\title{
Continuous Cauchy wavelet transform analyses of EXAFS spectra: A qualitative approach.
}

\author{
Manuel Munoz, Pierre Argoul, François Farges
}

\section{To cite this version:}

Manuel Munoz, Pierre Argoul, François Farges. Continuous Cauchy wavelet transform analyses of EXAFS spectra: A qualitative approach.. American Mineralogist, 2003, 88, pp.694-700. 10.2138/am2003-0423 . hal-00101932

\section{HAL Id: hal-00101932 \\ https://hal.science/hal-00101932}

Submitted on 28 Sep 2006

HAL is a multi-disciplinary open access archive for the deposit and dissemination of scientific research documents, whether they are published or not. The documents may come from teaching and research institutions in France or abroad, or from public or private research centers.
L'archive ouverte pluridisciplinaire HAL, est destinée au dépôt et à la diffusion de documents scientifiques de niveau recherche, publiés ou non, émanant des établissements d'enseignement et de recherche français ou étrangers, des laboratoires publics ou privés. 


\title{
Continuous Cauchy wavelet transform analyses of EXAFS spectra:
}

\section{A qualitative approach}

\author{
Manuel Muñoz ${ }^{1, *}$, Pierre Argoul $^{2}$ and François Farges ${ }^{1,3}$ \\ ${ }^{1}$ Laboratoire des Géomatériaux, Université de Marne-La-Vallée, CNRS FRE 2455, \\ 77454 Marne-La-Vallée cedex 2, France. \\ ${ }^{2}$ Laboratoire Analyse des Matériaux et Identification, Unité Mixte ENPC-LCPC, \\ 77455 Marne-La-Vallée cedex 2, France. \\ ${ }^{3}$ Department of Geological and Environmental Sciences, Stanford University, \\ CA 94305-2115, USA.
}

An article submitted for publication to "American Mineralogist"

version 8.0 - October 2002

Keywords:

NEW TECHNIQUE: Cauchy Wavelet

XAS (XAFS, XANES): EXAFS

GLASS PROPERTIES: Speciation

CRYSTAL STRUCTURE: Thorite Zircon

* Corresponding author: Manuel Muñoz

Laboratoire des Géomatériaux

Université de Marne-La-Vallée_munoz@oreka.com

Cité Descartes - Champs S/Marne

TEL +33149329103

77454 Marne-La-Vallée Cedex, France.

FAX +33149329137 


\section{AbSTRACT}

To better understand the extended x-ray absorption fine structure (EXAFS)

spectroscopic information obtained for complex materials such as those encountered in Earth sciences, we propose to use the Continuous Cauchy Wavelet Transform (CCWT). Thanks to this method, the EXAFS spectra can be visualized in three-dimension: the wavevector $(k)$, the interatomic distance uncorrected for phase-shifts $\left(R^{\prime}\right)$ and the CCWT modulus (corresponding to the continuous decomposition of the EXAFS amplitude terms). Consequently, more straightforward qualitative interpretations of EXAFS spectra can be performed, even when spectral artifacts are present, such as multiple-scattering features, multi-electronic excitations or noise. More particularly, this method provides important information concerning the $k$ range of each EXAFS contribution, such as next nearest-neighbors identification. To illustrate the potential of CCWT analyses applied to EXAFS spectra, we present first the experimental and theoretical spectra obtained for well-crystallized minerals, thorite and zircon, at the $\mathrm{Th}_{\mathrm{III}}$, and Zr K-edges, respectively. Then, we present the CCWT analyses of EXAFS spectra collected for amorphous materials of geochemical and environmental interests, including a sodium trisilicate glass and an aqueous chloride solution, at the Mo K-, and $\mathrm{Au} \mathrm{L}_{\mathrm{III}}$-edges, respectively. 


\section{INTRODUCTION}

Extended x-ray absorption fine structure (EXAFS) spectroscopy is a powerful tool for investigating the short and medium range environment around a selected absorbing atom in materials like minerals, glasses and solutions (see Brown et al. 1988; Brown et al. 1995; Henderson et al. 1995 for reviews). Structural parameters (i.e., interatomic distances, coordination numbers, Debye-Waller factors, etc.) can be accurately yielded thanks to the analysis and the modeling of the EXAFS spectra. Notably, the recent development of ab initio packages, like for example FEFF (Rehr et al. 1992), GNXAS (Filipponi et al. 1995; Filipponi and Di Cicco 1995) or EXCURVE (Binsted and Hasnain 1996), allows an efficient approach of the data reduction, particularly for crystalline compounds. However, the interpretations concerning complex systems (i.e., aperiodic structures, adsorption mechanisms, samples under extreme conditions, etc.) are generally more difficult. In such systems, Fourier transform (FT) analyses (Lytle et al. 1975) help to visualize the various shells of neighboring atoms surrounding the central atom probed. In addition, inverse FT filtering $\left(\mathrm{FT}^{-1}\right)$ is used to extract the different components to the EXAFS signal, $\chi(k)$ (i.e., the various pseudo-periodic contributions corresponding to a specific shell of neighbors around the central atom; Lytle et al. 1975). The theoretical equation of the EXAFS signal can be written as (Sayers et al. 1970; Stern et al. 1975): 


$$
\chi(k) \approx \sum_{j} S_{i}(k) \frac{N_{j}}{k R_{j}^{2}}\left|f_{j}(k, \pi)\right| e^{-2 R_{j} / \lambda_{j}(k)} e^{-2 \sigma{ }_{j}^{2} k^{2}} \sin \left(2 k R_{j}+\sum \phi_{i j}(k)\right)
$$

in which $S_{i}$ is the amplitude reduction factor for the total central atom loss. For each shell of neighboring atoms $j, N_{j}$ is the number of backscattering atoms, $R_{j}$ is the average distance between the central and the backscattering atoms, $\left|f_{j}(k, \pi)\right|$ is the effective curved-wave backscattering amplitude function, $\sigma_{j}^{2}$ is the Debye-Waller factor, $\lambda_{j}$ is the photoelectron mean free path, and $\sum \phi_{i j}(k)$ is the sum of the phase-shift functions including the backscattering phase-shifts of the central and neighboring atoms, as well as some anharmonic contributions related to thermal vibrations (Stern and Heald 1983; Teo 1986; Stern et al. 1991). Also, the wavevector $k$ is defined by the relation: $k=\sqrt{\frac{2 m_{e}}{h^{2}\left(E-E_{0}\right)}}$, in which $m_{e}$ is the electron mass, $h$ is Planck's constant, $E_{0}$ is the threshold energy of the absorption edge and $E$ is the absolute energy.

Based on Equation 1, the type of backscattering neighbors is determined, among others, based on the shape of the backscattering amplitude function, $\left|f_{j}(k, \pi)\right|$; the atomic number being determined at $\mathrm{Z} \pm 10$ (see Teo 1986). However, instead of examining each $\mathrm{FT}^{-1}$ independently from each other (with all the problems related to the possible overlap between close shells of neighbors), it would be more efficient to observe all the EXAFS contributions 
at once, by plotting them in the reciprocal $(k)$ and direct $\left(R^{\prime}\right)$ spaces simultaneously $\left(R^{\prime}\right.$ being the phase-shifts uncorrected distance).

To achieve this, we propose to use the continuous wavelet analysis (Louis et al. 1997; Torrésani 1999) to decompose the analyzed signals in the $\left(k, R^{\prime}\right)$ space in order to perform more straightforward interpretations of EXAFS spectra collected for samples of mineralogical, geochemical, and environmental interests. These last authors showed that the continuous wavelet analysis is an efficient method to analyze some "frequency-modulated" signals. The concept of wavelet analysis was first introduced by the geophysicist Jean Morlet in the beginning of the 1970's, based on early work on "time-frequency" Haar-Gabor decompositions (see Haar 1910). In petroleum exploration, wavelet analyses are widely used to analyze seismic signals in order to help discovering deep oil reservoirs (Morlet and Grossmann 1984; Goupillaud et al. 1984). Since that pioneering work, other types of wavelet analyses were developed for a broad range of applications like, for instance, video and audio compression (see Daubechies 1988 among many others). Applied to EXAFS spectroscopy, wavelet theory was first used to remove the atomic background (Shao et al. 1998), but also to reconstruct the radial distribution functions (Yamaguchi et al. 1999). In this study, we propose to use the continuous Cauchy wavelet transform (CCWT; Argoul and Le 2002) to decompose the EXAFS signal in the reciprocal and real spaces simultaneously. Then, EXAFS spectra can be visualized in three-dimension (3D): the wavevector $(k)$, the interatomic distance uncorrected for phase-shifts $\left(R^{\prime}\right)$ and the CCWT modulus. Consequently, the CCWT 
Muñoz et al. (2002) CCWT analyses of EXAFS spectra - Page 6

analysis provides an informative 3D view of the $\left(k, R^{\prime}\right)$ dependency of each EXAFS component; such information being particularly useful for the identification of the various contributions composing an EXAFS spectrum. In this study, we present several examples about transition elements in various geomaterials: two crystals, a silicate glass and an aqueous solution. 


\section{Continuous Cauchy Wavelet Transform}

\section{Numerical Description}

The continuous wavelet transform of a given frequency-modulated signal $\chi(k)$ is defined as follows (Chui 1992; Torrésani 1999):

$$
T_{\psi}[\chi](b, a)=\left\langle\chi(k), \psi_{(b, a)}(k)\right\rangle=\frac{1}{a} \int_{-\infty}^{+\infty} \chi(k) \bar{\psi}\left(\frac{k-b}{a}\right) d k
$$

in which $\left\langle\chi(k), \psi_{(b, a)}(k)\right\rangle$ is the scalar-product of the two functions $\chi(k)$ and $\Psi_{(b, a)}(k)$. Applied to EXAFS spectra, $\chi(k)$ represents the EXAFS signal (Eq. 1), usually $k^{3}$-weighted and multiplied by a smooth apodisation window. The function $\psi_{(b, a)}(k)$ represents a so-called "family" of wavelets, characterized by a constant shape and variable sizes ( $\bar{\psi}$ denotes the conjugate of $\psi$ ). Also, the parameters $b$ and $a$ are related to the $k$ - and $R^{\prime}$-spaces, respectively. The variable $b$ corresponds to any value of the $k$-vector, whereas the variable $a$ (so-called "scale-parameter") is here defined as $n / 2 R^{\prime}$, in which $n$ is a parameter related to the type of wavelet used (see below for details). 
The numerical computation of the continuous wavelet transform is based on fast Fourier transform algorithm (Cooley method). Then, following Argoul et al. (1998), Equation 2 can be written:

$$
T_{\psi}[\chi](b, a)=\frac{1}{\pi} \int_{0}^{+\infty} \hat{\chi}\left(R^{\prime}\right) \overline{\hat{\psi}}\left(2 a R^{\prime}\right) e^{2 i b R^{\prime}} d R^{\prime}
$$

in which $i$ is the complex number. Also the Fourier transform $\hat{\chi}\left(R^{\prime}\right)$ of the signal $\chi(k)$ is defined as:

$$
\hat{\chi}\left(R^{\prime}\right)=\int_{0}^{+\infty} \chi(k) e^{-2 i k R^{\prime}} d k
$$

Consequently, Equation 3 shows that, for a given value for $a$, the wavelet transform $T_{\psi}[\chi](b, a)$ can be seen as an inverse Fourier transform of the function $\hat{\chi}\left(R^{\prime}\right)$ convoluted by the function $\overline{\hat{\psi}}\left(2 a R^{\prime}\right)$. Thus, the wavelet transform being a complex matrix, the amplitude and the phase terms of the analyzed signal are calculated from the real and the imaginary parts.

In Equation 3, the function $\psi$ is called "mother" or "analyzing" wavelet ( $\overline{\hat{\psi}}$ being the conjugate of the FT of $\psi$ ). In this study, we chose to use the complex-valued Cauchy wavelet 
of order $n(n \geq 1)$. The wavelet transform is then called continuous Cauchy wavelet transform (CCWT). The Cauchy wavelet $\psi_{n}(k)$ and its FT $\hat{\psi}_{n}\left(R^{\prime}\right)$ are, respectively, defined as (Argoul and Le 2002):

$$
\psi_{n}(k)=\left(\frac{i}{k+i}\right)^{n+1} \quad \hat{\psi}_{n}\left(R^{\prime}\right)=2 \pi \frac{R^{\prime n}}{n !} e^{-R^{\prime}} H\left(R^{\prime}\right)
$$

in which $H\left(R^{\prime}\right)$ is the Heaviside step function. Also, the Cauchy-parameter $n$ controls the resolutions, $\Delta k$ and $\Delta R^{\prime}$, of the CCWT in the $k$ - and $R^{\prime}$-spaces, respectively. Following Argoul and Le (2002), the distribution of resolutions is defined by:

$$
[k-\Delta k, k+\Delta k] \times\left[R^{\prime}+\Delta R_{1}^{\prime}, R+\Delta R_{2}^{\prime}\right]
$$

with a symmetric shape in the reciprocal space:

$$
\Delta k=\frac{1}{R^{\prime}}\left(\frac{n}{2 \sqrt{2 n-1}}\right)
$$

and an asymmetric shape in the direct space:

$$
\Delta R_{1}^{\prime}=R^{\prime}\left(\frac{1}{2 n}-\frac{\sqrt{2 n+1}}{2 n}\right) \quad \Delta R_{2}^{\prime}=R^{\prime}\left(\frac{1}{2 n}+\frac{\sqrt{2 n+1}}{2 n}\right)
$$


In Equations 7 and 8 , note that $\Delta k$ and $\Delta R^{\prime}$ are inversely proportional. Moreover, $\Delta k$ and $\Delta R^{\prime}$ are constrained by the Heisenberg inequality: $\Delta k \cdot \Delta R^{\prime} \geq 1 / 4$ (Chui 1992). Thus, for a given $n$-value, $\Delta R^{\prime}$ is small and $\Delta k$ is high for low $R^{\prime}$-values. Reciprocally, $\Delta R^{\prime}$ is high and $\Delta k$ is small for high $R^{\prime}$-values.

The Cauchy mother wavelet has been chosen because of its "progressive" properties, which means that its FT vanishes for $R^{\prime}<0$ (due to the Heaviside step function). An example of the application of the Cauchy wavelet for the two-dimensional continuous wavelet analysis is the determination of "hidden" symmetries in the crystal structure of quasi-crystalline alloys (see Antoine et al. 1999a, 1999b). Moreover, the use of a complex-valued wavelet (by opposition to a real one, i.e., $\in \mathbb{R}$ ) simplifies the numerical computation thanks to its real FT, and is well suited for analyzing frequency-modulated signals (Torrésani 1999; Argoul et al. 2002).

As the asymptotic properties are respected for the analyzed signal (i.e., the phase term is varying faster than the amplitude term; Argoul et al. 1998), the CCWT modulus tend to concentrate all the information related to EXAFS contributions in the $\left(k, R^{\prime}\right)$ space, near a series of curves called "CCWT ridges" (see Carmona et al. 1997; Carmona et al. 1998). For that reason, the analyzed EXAFS signal is generally $k^{3}$-weighted and multiplied by an 
apodisation window before computation. Then, according to Carmona et al. (1998), Equation 3 can be written as:

$$
T_{\psi_{n}}[\chi](b, a) \approx \frac{1}{2} \sum_{j} \hat{\psi}_{n}\left(a \cdot \Phi_{j}^{\prime}(b)\right) \mathrm{A}_{j}(b) e^{i \Phi_{j}(b)}
$$

in which $\mathrm{A}_{j}(b)$ represents the EXAFS amplitude term $\left(S_{i}(k) \frac{N_{j}}{k R_{j}^{2}}\left|f_{j}(k, \pi)\right| e^{-2 R_{j} / \lambda_{j}(k)} e^{-2 \sigma_{j}^{2} k^{2}}\right)$, and $\Phi_{j}(b)$ the EXAFS phase term $\left(2 k R_{j}+\sum \phi_{i j}(k)\right)$ for each $j^{\text {th }}$ pseudo-periodic contribution related to a shell of backscattering neighbors (see Eq. 1 ); $\Phi_{j}^{\prime}(b)$ being the derivative of $\Phi_{j}(b)$.

In the $(b, a)$ space of the CCWT, each $j^{\text {th }}$ ridge $a_{j}(b)$ is defined as $n / \Phi_{j}^{\prime}(b)$ (Carmona et al. 1997; Carmona et al. 1998). Therefore, when the scale-parameter $a$ is localized on a given $j^{\text {th }}$ ridge (i.e., $\left.a=a_{j}(b)\right)$, the CCWT phase corresponds to $\Phi_{j}(b)$, and the CCWT modulus becomes:

$$
\left|T_{\psi_{n}}[\chi]\right|\left(b, a=a_{j}(b)\right) \approx \frac{1}{2} \hat{\psi}_{n}(n) \mathrm{A}_{j}(b)
$$

Consequently, on each $j^{\text {th }}$ ridge observed, the CCWT modulus provides the amplitude term $\mathrm{A}_{j}(b)$ of a given EXAFS signal contribution (to within a wavelet-defined constant $\left.\frac{1}{2} \hat{\psi}_{n}(n)\right)$. 
In this paper, each EXAFS spectrum was previously $k^{3}$-weighted, and multiplied by the Kaiser-Bessel window with a Kaiser-Bessel parameter of 4 (Bonnin et al. 1985). Moreover, all the CCWT presented were calculated with $n=200$, and typically, 400 values were used for the scale-parameter $a$ (corresponding to the number of pixels in the $R^{\prime}$ dimension). Therefore, for $R^{\prime}$-values around $2 \AA$, the resolutions in the $k$ - and $R^{\prime}$-spaces are, respectively, $\Delta k \approx \pm 2.5 \AA^{-1}$ and $\Delta R^{\prime} \approx \pm 0.1 \AA$, whereas around $6 \AA, \Delta k \approx \pm 0.8 \AA^{-1}$ and $\Delta R^{\prime} \approx$ $\pm 0.3 \AA$.

\section{Application to EXAFS spectroscopy}

This study focuses mostly on qualitative analyses of EXAFS spectra, based on the study of the CCWT-filtered EXAFS amplitude terms. Teo (1986) has shown that the variations in the amplitude of a given normalized EXAFS spectrum are importantly affected by the backscattering amplitude functions of the neighboring atoms $\left(\left|f_{j}(k, \pi)\right|\right.$ in Eq. 1); this last one being related to the number of (repulsive) electrons in the electronic cloud of the backscattering neighbors. Therefore, atoms having high atomic numbers are more efficient backscatterers at high $k$-values, in contrast to atoms having lower atomic numbers, which are more efficient backscatterers at lower $k$-values. Consequently, since the CCWT modulus is a decomposition of each $j^{\text {th }}$ EXAFS amplitude term in the $R^{\prime}$-space (see Eq. 10), the interpretation of a CCWT calculation is here essentially based on the graphical analysis of its 
modulus. Similarly, the CCWT phase corresponds to the decomposition of each $j^{\text {th }}$ EXAFS phase term in the $R^{\prime}$-space. However, despite the CCWT phase contains valuable information, the qualitative interpretation of the atomic neighboring shells is much less direct (see Eq. 1), and its analysis will be presented in a forthcoming study.

By simply comparing the relative variations for each ridge of the CCWT modulus, we obtain qualitative information about the various contributions to the EXAFS spectrum. To illustrate and validate the use of CCWT analysis applied to EXAFS spectra, we will present the study of $\mathrm{Th}$ and $\mathrm{Zr}$ in two crystalline model compounds: thorite and zircon, respectively. Then, we will apply the CCWT method to the analysis of experimental EXAFS spectra collected for molybdenum and gold in two aperiodic systems: a sodium trisilicate glass and an aqueous chloride solution. 


\section{RESULTS}

\section{Crystalline thorite}

A synthetic crystalline $\alpha$-thorite $\left(\mathrm{ThSiO}_{4}\right)$ has been investigated using EXAFS spectroscopy at the $\mathrm{Th}_{\mathrm{III}}$-edge (see Farges and Calas 1991). Figure 1 presents the EXAFS spectrum (Fig. 1a) with the corresponding FT (Fig. 1b) and CCWT (Fig. 1c) analyses. On the FT spectrum, each peak is identified according to the crystal structure refinement of Taylor and Ewing (1978). In agreement with this study, four main contributions are observed: (a) 8 oxygen first-neighbors $(\approx 2.0 \AA$ on the FT; at an averaged Th-O distance of $2.41 \AA$ ), (b) $2 \mathrm{Si}$ second-neighbors $(\approx 2.9 \AA$ on the $\mathrm{FT} ;\langle\mathrm{Th}-\mathrm{Si}>=3.16 \AA$ ), (c) a mixed shell with $4 \mathrm{Si}$ and $4 \mathrm{Th}$ third-neighbors $(\approx 3.8 \AA$ on the FT; $\langle\mathrm{Th}-\mathrm{Si} / \mathrm{Th}>=3.90 \AA$ ) and finally, (d) a last shell arising essentially from 12 Th next nearest-neighbors $(\approx 5.9 \AA$ on the FT; $<\mathrm{Th}-\mathrm{Th}>=5.93-5.95 \AA)$. Each of these four contributions can be associated to a distinct ridge on the CCWT modulus, labeled, respectively, $\mathrm{O}, \mathrm{Si}, \mathrm{Si} / \mathrm{Th}$ and $\mathrm{Th}$ on Figure 1c. On this figure, the EXAFS amplitude terms arising from oxygen, silicon and thorium present well different shapes along the $k$ space. The 3D representation of the CCWT modulus (Fig. 2) provides a complementary view of the shape of each EXAFS amplitude term. In that case, Figures 1c and 2 show that the oxygen first-neighbors contribute significantly for a $k$-range of 2-14 $\AA^{-1}$, whereas $\mathrm{Si}$ and Th next nearest-neighbors are significant for $k$-ranges of 7-13 $\AA^{-1}$ and $11-16 \AA^{-1}$, respectively. 
To further understand the CCWT analyses presented on Figures 1c and 2, ab initio EXAFS calculations of thorite at the $\mathrm{Th}_{\mathrm{III}}$-edge were carried out using the FEFF 7.02 package (Rehr et al. 1992; Ankudinov et al. 1998). The calculations were based on the crystal structure refinement of Taylor and Ewing (1978). To calculate theoretical EXAFS spectra, we used default settings for the atomic pair potential (i.e., Hedin-Lunqvist), and automatic overlapping of the muffin-tin radii (AFOLP option). Debye-Waller factors were adjusted to match the experimental spectra, and multiple-scattering (MS) paths were included in the calculation. The CCWT analyses of the experimental spectrum (Fig. 3a) and its theoretical counterpart (Fig. 3b) are in good agreement with each other. Also, each of the four EXAFS contributions (described above) can be identified, according to their EXAFS amplitude terms calculated by FEFF 7.02 (Fig. 3c). For instance, the EXAFS contribution related to the 8 oxygen first-neighbors is centered around $6 \AA^{-1}$ on the CCWT modulus (Fig. 3b), which is consistent with the maximum of its theoretical amplitude term (Fig. 3c). Similar agreements can be found for the more distant contributions ( $\mathrm{Si}$ and Th next nearest-neighbors are centered around 9 and $15 \AA^{-1}$, respectively).

The ridge located near $1.2 \AA$ on Figure 3a can be attributed to a spectral artifact because no atom can be located between the central absorbing thorium and the oxygen firstneighbors. The origin of such contribution is most likely related to multi-electronic excitation features (see Filipponi et al. 1991). Indeed, these features generate discontinuities in the atomic background of the EXAFS signal (see Campbell et al. 2002), and therefore, generate 
some "low frequencies" in the $R^{\prime}$-space (see Solera et al. 1995). According to Farges et al. (2000), up to four suspected transitions (located near 2.5, 4.0, 5.5 and $10 \AA^{-1}$ ) could be identified in the EXAFS of thorite at the Th $\mathrm{L}_{\mathrm{III}}$-edge. However, because FEFF 7.02 do not calculate multi-electronic excitations, these features are not observed on Figure 3b. Also, FEFF calculations suggested that MS contributions are less intense as compared to the single scattering ones. Consequently, their amplitudes are not clearly distinguishable on the CCWT modulus (Fig. 3b).

\section{Thorite vs. zircon}

Because thorite and zircon are isostructural (I $4_{1} / \mathrm{amd}$ ), it is possible to highlight the effect of the cationic substitution, $\mathrm{Th} \leftrightarrow \mathrm{Zr}$, on the CCWT modulus. The EXAFS spectrum of crystalline zircon $\left(\mathrm{ZrSiO}_{4}\right)$ at the $\mathrm{Zr} \mathrm{K}$-edge has been calculated using FEFF 7.02, according to the crystal structure refinement of Robinson et al. (1971). The calculation has been performed in the same conditions than previously. Also, MS paths of the photoelectron were included in the calculation. However, their amplitudes were relatively low. Therefore, the main contributions to the EXAFS spectrum essentially arise from the single-scattering paths. CCWT analyses of theoretical EXAFS spectra are shown on Figures $4 \mathrm{a}$ and $4 \mathrm{~b}$ for thorite and zircon, respectively. Both images present large similarities concerning, in particular, the maximums of the ridges related to oxygen and silicon (labeled $\mathrm{O}$ and $\mathrm{Si}$ on Figures 4a and $4 \mathrm{~b})$, along the $k$-axis. In contrast, the two ridges related to zirconium on Figure $4 \mathrm{~b}(\langle\mathrm{Zr}-\mathrm{Zr}\rangle=$ 
3.63 and $5.55 \AA$; Robinson et al. 1971) show maximums near $11 \AA^{-1}$, whereas those arising from thorium (<Th-Th> = 3.90 and $5.95 \AA ̊$; Taylor and Ewing 1978) on Figure 4a are localized near 13-14 $\AA^{-1}$. That difference can be attributed to the different atomic numbers of Th and $\mathrm{Zr}$ (90 and 40, respectively), traducing their different backscattering amplitude functions (Eq. 1). 


\section{DiscuSSION}

\section{Molybdenum in a sulfur-bearing silicate glass}

A sodium trisilicate glass $\left(\mathrm{Na}_{2} \mathrm{Si}_{3} \mathrm{O}_{7}\right)$ doped with 2000 ppm of molybdenum was synthesized under controlled oxygen and sulfur fugacities, of $10^{-10.2}$ and $10^{-1.6} \mathrm{~atm}$, respectively (Siewert et al. 2002). The experimental EXAFS spectrum was collected at the Mo K-edge, up to $14 \AA^{-1}$ (Fig. 5a). Its FT spectrum (Fig. 5b) presents two peaks having approximately the same height, and centered around 1.4 and $1.8 \AA$. Those two peaks are related to a double shell of first-neighbors. However, using a simple FT analysis it is not directly possible to determine if they arise from two oxygen shells, two sulfur shells or a mixture of both. Moreover, the two contributions are too close to each other $\left(\Delta R^{\prime} \approx 0.4 \AA\right)$ to be filtered by $\mathrm{FT}^{-1}$ method, and only a double shell fit can help to identify them. In contrast, with only one CCWT calculation, a 3D representation of the modulus (Fig. 5c) clearly shows well different shapes for the ridges of those two contributions. Therefore, two different types of atoms are surrounding the central molybdenum. Moreover, Figure 5c shows that the closest contribution (around $1.4 \AA$ ) is centered on relatively low $k$-values as compared to the more distant one (around $1.8 \AA$ ). Consequently, because only two types of anions, having well different atomic numbers, are present in this glass (i.e., oxygen, $\mathrm{Z}=8$ and sulfur, $\mathrm{Z}=$ 16), one can infer that the closest contribution is most likely related to oxygen and the other one is most likely related to sulfur (see the previous sections for details). Those results 
indicate that molybdenum forms oxy-sulfide complexes in sodium trisilicate glasses, helping to understand its transport properties in magmatic systems (see Siewert et al. 2002 for details).

\section{Gold in an aqueous chloride solution}

An aqueous chloride solution equilibrated at $\mathrm{pH}=9.2$ and containing $0.01 \mathrm{M}$ of gold was investigated using EXAFS spectroscopy at the Au $\mathrm{L}_{\mathrm{III}}$-edge (Farges et al. 1993). The EXAFS spectrum, as well as FT and CCWT analyses are presented on Figures $6 \mathrm{a}, 6 \mathrm{~b}$ and $6 \mathrm{c}$, respectively. Here again, a double shell of first-neighbors surrounds the absorbing atom. Two peaks are present on the FT (Fig. 6b), the closest contribution (around $1.7 \AA$ ) being approximately two times higher than the other one (around 2.0 ̊). The CCWT modulus (Fig. 6c) presents two different shapes for each EXAFS amplitude term, suggesting the presence of two different types of anions around the gold central atom. The closest ridge $(\approx 1.7 \AA)$ is centered near $6 \AA^{-1}$, whereas the other one $(\approx 2.0 \AA)$ is centered near $8.5 \AA^{-1}$. As for the previous example, we can infer that the closest contribution is most likely related to oxygen atoms because of its relatively low atomic number $(\mathrm{Z}=8)$, whereas the other one is most likely related to chlorine atoms $(Z=17)$. This result is in excellent agreement with the study of Peck et al. (1991), showing the presence of $\mathrm{Au}(\mathrm{OH})_{2} \mathrm{Cl}_{2}$ moieties, with gold atom located in a square planar polyhedra. Also, the study of Farges et al. (1993) suggests the presence of two oxygen atoms located at $1.97 \AA$ and two chlorine atoms located at $2.28 \AA$ from the gold central atom. 
Figure 6c presents a "gray spot" localized between 3 and $4 \AA$ in the $R^{\prime}$-space, and centered below the oxygen one in the $k$-space, from around 2 to $8 \AA^{-1}$. However, the squareplanar geometry around gold (i.e., $\mathrm{AuX}_{4}$ polyhedra, with $\mathrm{X}=\mathrm{O} / \mathrm{Cl}$ ) importantly involves the photoelectron MS paths of order 3 and 4, generating three main contributions between 3.4 and 4.0 $\AA$ (Berrodier et al. 1999). Therefore, this "gray spot", corresponding to a combination of CCWT ridges, can be reasonably related to MS contributions.

In addition, when $k$-values increase, the "high-frequencies" related to the noise interfere progressively with the "low-frequencies" (i.e., the low $R^{\prime}$-values) arising from the different contributions to the EXAFS signal. Indeed, the EXAFS spectrum on Figure 6a shows an increasing noise level with increasing $k$-values. In the same time, the CCWT modulus (Fig. 6c) highlights the noise domain, which is delimitated from the structural contributions and from the MS features (occurring below $10 \AA^{-1}$ ), as shown by the solid line. Consequently, the CCWT modulus provides an interesting representation, where structural contributions, MS features and noise are located in well different regions in the $\left(k, R^{\prime}\right)$ space. 


\section{CONCLuSions}

By providing a continuous decomposition of an EXAFS spectrum in the reciprocal and real spaces simultaneously, the CCWT analysis is presented here as a useful complementary tool for EXAFS data reduction applied to complex earth materials. Thanks to that method, any EXAFS spectrum can be visualized in three-dimension: the wavevector $(k)$, the interatomic distance uncorrected for phase-shifts $\left(R^{\prime}\right)$ and CCWT modulus (corresponding to the continuous decomposition of the EXAFS amplitude terms). Consequently, because the $\left(k, R^{\prime}\right)$ decomposition is obtained with only one calculation, the qualitative interpretations of EXAFS spectra, based on comparisons between the different amplitude terms, are particularly straightforward. In order to validate the efficiency of that method, some theoretical ab initio EXAFS calculations were performed on well-known model compounds. The combination of FEFF 7.02 calculations and CCWT analyses validated the correlation between, the theoretical amplitude terms of the single-scattering EXAFS contributions, and the ridges of the CCWT modulus. In addition, it was possible to localize, in well different regions of the $\left(k, R^{\prime}\right)$ space, different EXAFS contributions like single-scattering, multiple-scattering, multi-electronic excitations and noise. Further studies of CCWT analyses applied to EXAFS spectra are underway, including the use of CCWT phase terms in order to precisely characterize and extract some quantitative anharmonic information from each EXAFS contribution. 


\section{ACKNowledgments}

We thank the staff of LURE (Orsay, France) and SSRL (Stanford, USA) for help in data collection, as well as Julia Peck (formally at Stanford University) and Ralf Siewert (formally at Université de Marne-La-Vallée) for providing samples. Comments on the manuscript made by Stéphanie Rossano (Université de Marne-La-Vallée) were greatly appreciated. 


\section{REFERENCES}

Ankudinov, A.L., Ravel, B., Rehr, J.J., and Conradson, S.D. (1998) Real-space multiplescattering calculation and interpretation of X-ray-absorption near-edge structure. Physical Review B, 58, 7565-7576.

Antoine, J.-P., Jacques, L., and Twarock, R. (1999a) Wavelet analysis of a quasiperiodic tiling with fivefold symmetry. Physics Letters A, 261, 265-274.

Antoine, J.-P., Murenzi, R., and Vandergheynst, P. (1999b) Directional wavelets revisited: Cauchy wavelets and symmetry detection in patterns. Applied and Computational Harmonic Analysis, 6, 314-345.

Argoul, P. and Le, T.-P. (2002) Wavelet analysis of transient signals in civil engineering. In M. Frémond and F. Maceri, Eds., Novel approaches in civil engineering. Springer Publishers, In press.

Argoul, P., Yin, H.-P., and Guillermin, B. (1998) Use of the wavelet transform for the processing of mechanical signals. In P. Sas, Ed., Proceedings of ISMA23 International Conference on Noise and Vibration Engineering, 1, p. 329-336. Leuven, Belgium.

Berrodier, I., Farges, F., Benedetti, M., and Brown, G.E. Jr. (1999) Adsorption of Au ferrihydrites using Au- $\mathrm{L}_{\text {III }}$ edge XAFS spectroscopy. Journal of Synchrotron Radiation, $6,651-652$.

Binsted, N. and Hasnain, S. (1996) State-of-the-art analysis of whole X-ray absorption spectra. Journal of Synchrotron Radiation, 3, 185-196.

Bonnin, D., Calas G., Suquet H., and Pezerat H. (1985) Sites occupancy of $\mathrm{Fe}^{3+}$ in Garfield nontronite: a spectroscopic study. Physics and Chemistry of Minerals, 12, 55-64.

Brown, G.E. Jr., Calas, G., Waychunas, G.A., and Petiau, J. (1988) X-ray absorption spectroscopy: Applications in mineralogy and geochemistry. In F.C. Hawthorne, Ed., Spectroscopic Methods in Mineraloy and Geochemistry, 18, p. 431-512. Reviews in Mineralogy. 
Brown, G.E. Jr., Farges, F., and Calas, G. (1995) X-ray scattering and x-ray spectroscopy studies of silicate melts. In J.F. Stebbins, D.B. Dingwell and P.F. McMillan, Eds., Structure, Dynamics, and Properties of Silicate Melts, 32, p. 317-410. Reviews in Mineralogy.

Carmona, R., Hwang W.L., and Torrésani, B. (1997) Characterization of signals by the ridges of their wavelet transforms. IEEE Transactions on Signal Processing, 45, 2586-2590.

Carmona, R., Hwang W.L., and Torrésani, B. (1998) Practical time-frequency analysis. Gabor and Wavelet transforms with an implementation in S. In C.K. Chui, Ed., Wavelet Analysis and Its Applications, 9, p. 271-308. Academic Press.

Campbell, L., Hedin, L., Rehr, J.J., and Bardyszewski, W. (2002) Interference between extrinsic and intrinsic losses in x-ray absorption fine structure. Physical Review B, 65, 64107-64120.

Chui, C.K. (1992) An introduction to wavelets, p. 60-64. Academic Press, San Diego, London.

Daubechies, I. (1988) Orthonormal bases of compactly supported wavelets. Communication on Pure and Applied Mathematics, 41, 909-996.

Farges, F. and Calas, G. (1991) Structural analysis of alpha-radiation damage in zircon and thorite: A x-ray absorption study. American Mineralogist, 76, 60-73.

Farges, F., Sharps, J.A., and Brown, G.E. Jr. (1993) Local environment around gold (III) in aqueous chloride solutions: An EXAFS spectroscopy study. Geochimica et Cosmochimica Acta, 57, 1243-1252.

Farges, F., Harfouche, M., Petit, P.-E., and Brown, G.E. Jr (2000) Actinides in Earth materials: The importance of natural analogues. In Reich and Shuh, Eds., Workshop proceedings of Speciation, Techniques, and Facilities for Radioactive Materials at Synchrotron Light Sources, 2, p. 63-74. Grenoble, France.

Filipponi, A. and Di Cicco, A. (1995) X-ray absorption spectroscopy and n-body distribution functions in condensed matter (II): data-analysis and applications. Physical Review B, 52, 15135-15149. 
Filipponi, A., Di Cicco, A., Tyson, T.A., and Natoli, C.R. (1991) Ab initio modeling of x-ray absorption spectra. Solid State Communications, 78, 265-268.

Filipponi, A., Di Cicco, A., and Natoli, C.R. (1995) X-ray absorption spectroscopy and nbody distribution functions in condensed matter (I): theory. Physical Review B, 52, $15122-15134$.

Goupillaud, P., Grossmann, A., and Morlet, J. (1984) Cycle-octave and related transforms in seismic signal analysis. Geoexploration, 23, 85-102.

Haar, A. (1910) Zur theorie der orthogonalen funktionen-systeme. Mathematische Annalen, $69,331-371$.

Henderson, C.M.B., Cressey, G., and Redfern, S.A.T. (1995) Geological applications of synchrotron radiation. Radiation Physics and Chemistry, 45, 459-481.

Louis, A.K., Maaß, P., and Rieder, A. (1997) Wavelets theory and applications. Pure and applied mathematics, p. 1-35. John Wiley \& Sons Publishers.

Lytle, F.W., Sayers, D.E., and Stern, E.A. (1975) Extended X-ray-absorption fine-structure technique. II. Experimental practice and selected results. Physical Review B, 11, 48254835 .

Morlet, J. and Grossmann, A. (1984) A decomposition of hardy functions into square integrable wavelets of constant shape. SIAM Journal on Mathematical Analysis, 15, 723-736.

Peck, J.A., Tait, C.D., Swanson, B.I., and Brown, G.E. Jr. (1991) Speciation of aqueous gold(III) chlorides from ultraviolet/visible absorption and Raman/resonance Raman spectroscopies. Geochimica et Cosmochimica Acta, 55, 671-676.

Rehr, J.J., Zabinsky, Z.I., and Albers, R.C. (1992) High-order multiple scattering calculations of x-ray-absorption fine structure. Physical Review Letters, 69, 3397-3400.

Robinson, K., Gibbs, G.V., and Ribbe, P.H. (1971) The structure of zircon: A comparison with garnet. American Mineralogist, 56, 782-790.

Sayers, D.E., Lytle, F.W., and Stern, E.A. (1970) Point scattering theory of X-ray Kabsorption fine structure. Advances in X-ray analysis, 13, 248-271. 
Shao, X., Shao, L., and Zhao, G. (1998) Extraction of extended X-ray absorption fine structure information from the experimental data using the wavelet transform. Analytical Communications, 35, 135-137.

Siewert, R., Farges, F., Brown, G.E. Jr., Büttner, H., Guesdon, A., Lapeyre, C., Morin, G., and Calas, G. (2002) Structural environments of incompatible elements in silicate glass/melt systems: Part III. Influence of composition and oxygen fugacity on the local structure of molybdenum. Geochimica et Cosmochimica Acta, Submitted.

Solera, J.A., Garcia, J., and Proietti, M.G. (1995) Multielectron excitations at the L edges in rare-earth ionic aqueous solutions. Physical Review B, 51, 2678-2686.

Stern, E.A. and Heald, S.M. (1983) Basic principles and applications of EXAFS. Handbook on Synchrotron Radiation, p. 955-1014.

Stern, E.A., Sayers, D.E., and Lytle, F.W. (1975) Extended X-ray-absorption fine-structure technique. III. Determination of physical parameters. Physical Review B, 11, 48364846.

Stern, E.A., Livins, P., and Zhang, Z. (1991) Thermal vibration and melting from a local perspective. Physical Review B, 43, 8850-8860.

Taylor, M. and Ewing, R.C. (1978) The crystal structures of the $\mathrm{ThSiO}_{4}$ polymorphs: Huttonite and thorite. Acta Crystallographica B, 34, 1074-1079.

Teo, B.K. (1986) EXAFS: Basic Principles and Data Analysis (ninth edition). 349 p. Inorganic Chemistry Concepts, Springer, Berlin.

Torrésani, B. (1999) Time-frequency and time-scale analysis. In J. Byrnes, Ed., Signal Processing for Multimedia, p. 37-52.

Yamaguchi, K., Ito, Y., and Mukoyama, T. (1999) The regularization of the basic x-ray absorption spectrum fine structure equation via the wavelet-Galerkin method. Journal of Physics B: Atomic Molecular and Optical Physics, 32, 1393-1408. 


\section{Figure Captions}

FIGURE 1. EXAFS analysis of crystalline thorite at the thorium $\mathrm{L}_{\mathrm{III}}$-edge: (a) $k^{3}$ weighted experimental EXAFS spectrum; (b) FT-magnitude; (c) CCWT modulus showing the $\left(k, R^{\prime}\right)$ localization of each EXAFS contribution. The FT peaks and CCWT ridges are labeled according to the structure refinement of Taylor and Ewing (1978).

FIGURE 2. Three-dimensional view of the CCWT modulus calculated from the $\mathrm{Th}_{\mathrm{III}}{ }^{-}$ edge EXAFS spectrum presented on Figure 1, showing well different EXAFS amplitude terms (or ridges) for the different atomic shells.

FIGURE 3. Analysis of the Th $\mathrm{L}_{\mathrm{III}}$-edge EXAFS spectrum for crystalline thorite: (a) CCWT of the experimental spectrum; (b) CCWT of the theoretical spectrum (using FEFF 7.02); (c) path decomposition, based on FEFF 7.02 calculations.

FIGURE 4. Comparison of the CCWT modulus for crystalline thorite (a) and zircon (b): (bottom) $k^{3}$-weighted EXAFS spectra calculated with FEFF 7.02 at the $\mathrm{Th}_{\mathrm{III}}$-edge and the $\mathrm{Zr}$ K-edge for thorite and zircon, respectively; (top) CCWT analyses of the EXAFS spectra. The ridges on the CCWT modulus are labeled according to the structure refinement of Taylor and Ewing (1978) and Robinson et al. (1971) for thorite and zircon, respectively. 
FIGURE 5. (a) $k^{3}$-weighted EXAFS of the $\mathrm{Na}_{2} \mathrm{Si}_{3} \mathrm{O}_{7}$ glass (2000 ppm of Mo and $2 \mathrm{wt} \%$ of S), collected at the Mo K-edge; (b) FT analysis of the EXAFS spectrum; (c) threedimensional view of the CCWT modulus (localized between 0 and $3 \AA$ ) calculated from the EXAFS spectrum. The 3D graph highlights the presence of a mixed environment around Mo, related to oxy-sulfide complexes.

FIGURE 6. (a) $k^{3}$-weighted EXAFS spectrum collected at the $\mathrm{Au} \mathrm{L}_{\mathrm{III}}$-edge in an $\mathrm{Au}$ (0.01 M)-aqueous chlorine solution ( $\mathrm{pH}=9.2)$; (b) FT analysis of the EXAFS spectrum; (c) CCWT analysis of the EXAFS spectrum. "O" and "Cl" represent oxygen and chlorine neighboring shells, respectively. "MS" corresponds to the spot related to the multiplescattering effects arising from the first-neighbors. The solid line separates the MS features and the single-scattering contributions form the noise. 
FIGURE 1

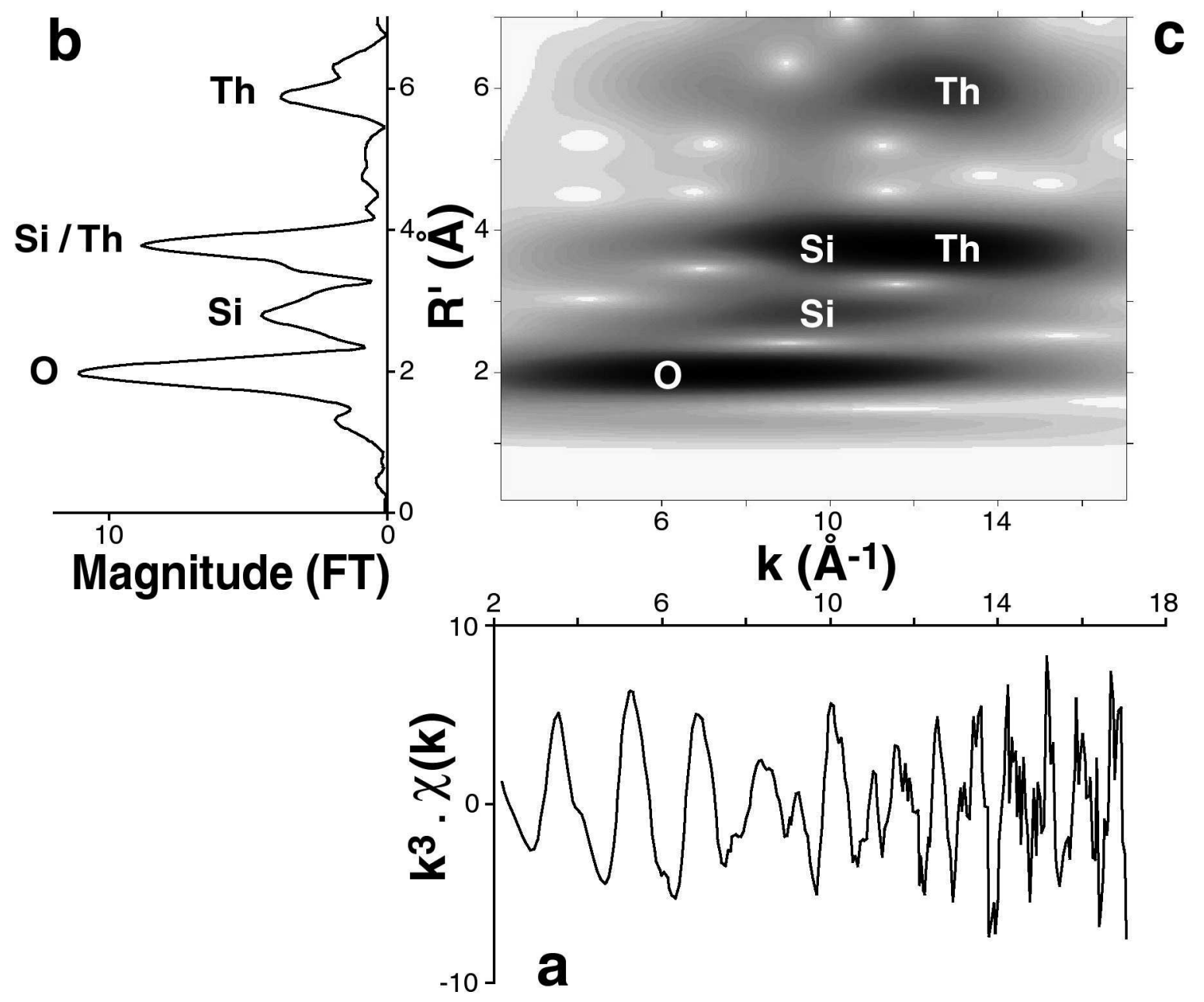


Muñoz et al. (2002) CCWT analyses of EXAFS spectra - Page 30

\section{FIGURE 2}

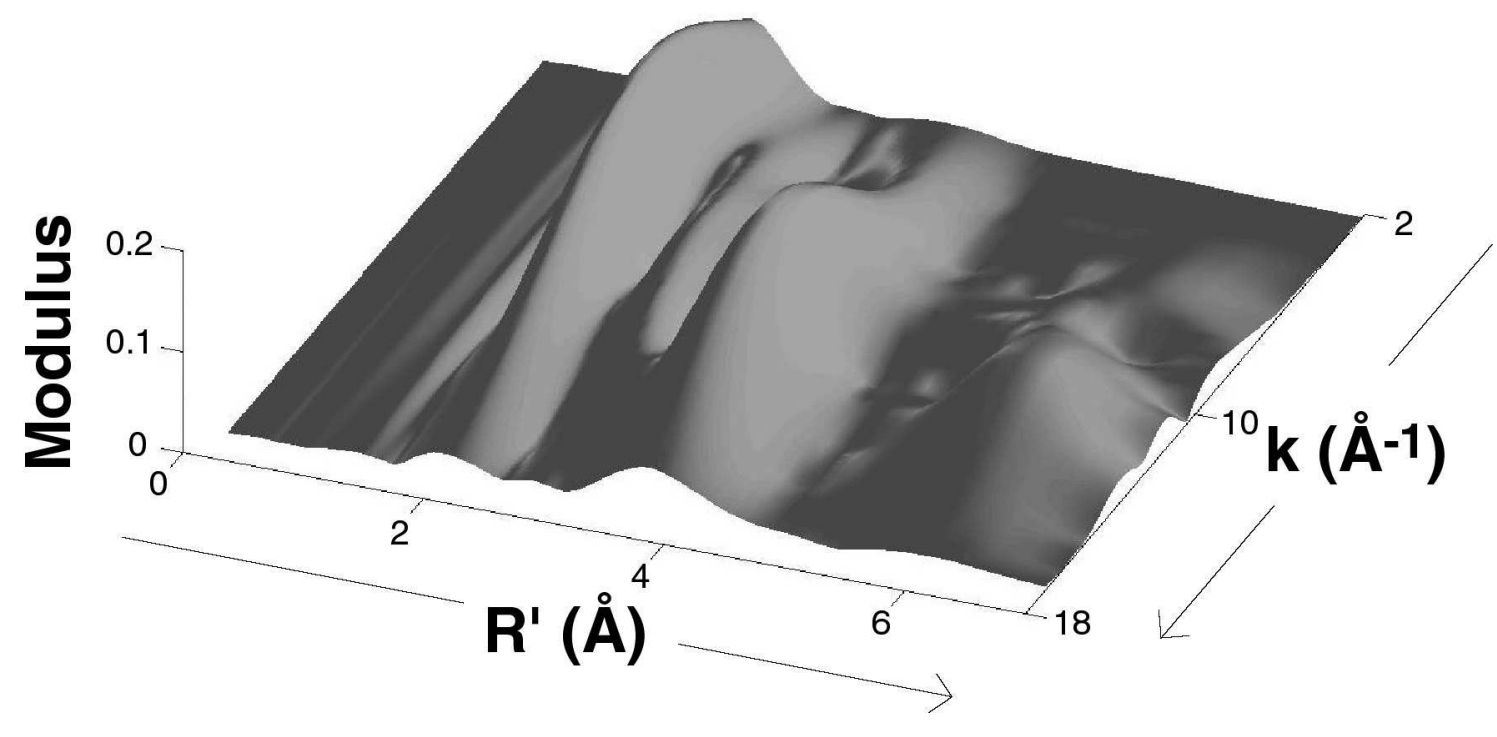


Muñoz et al. (2002) CCWT analyses of EXAFS spectra - Page 31

FIGURE 3
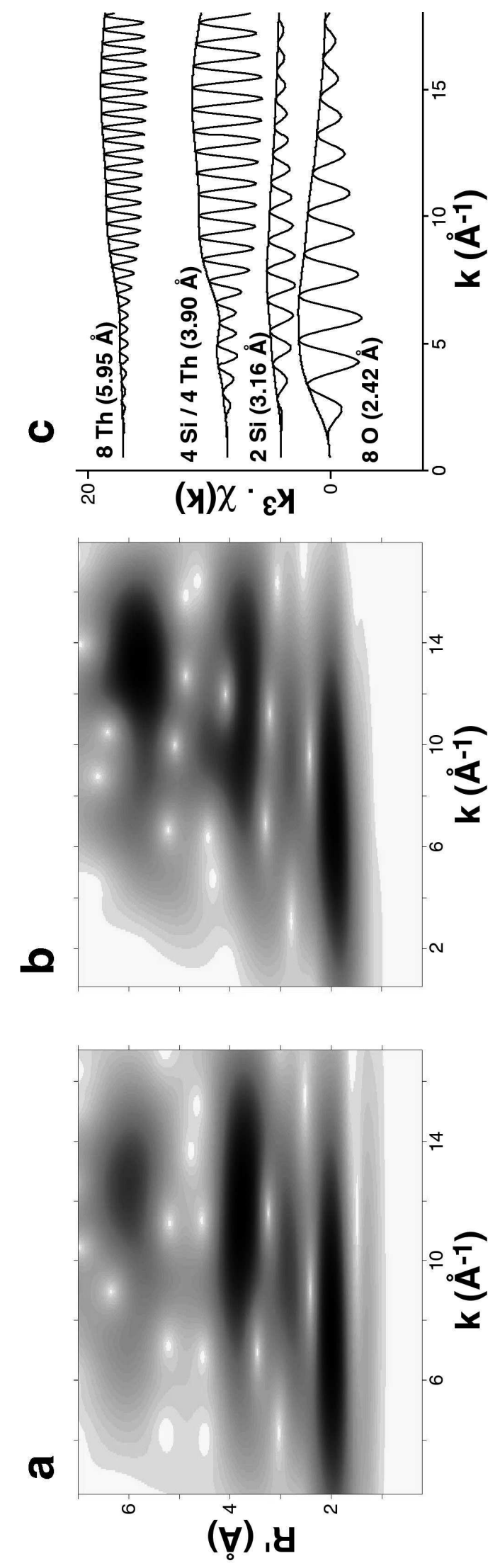
FIGURE 4

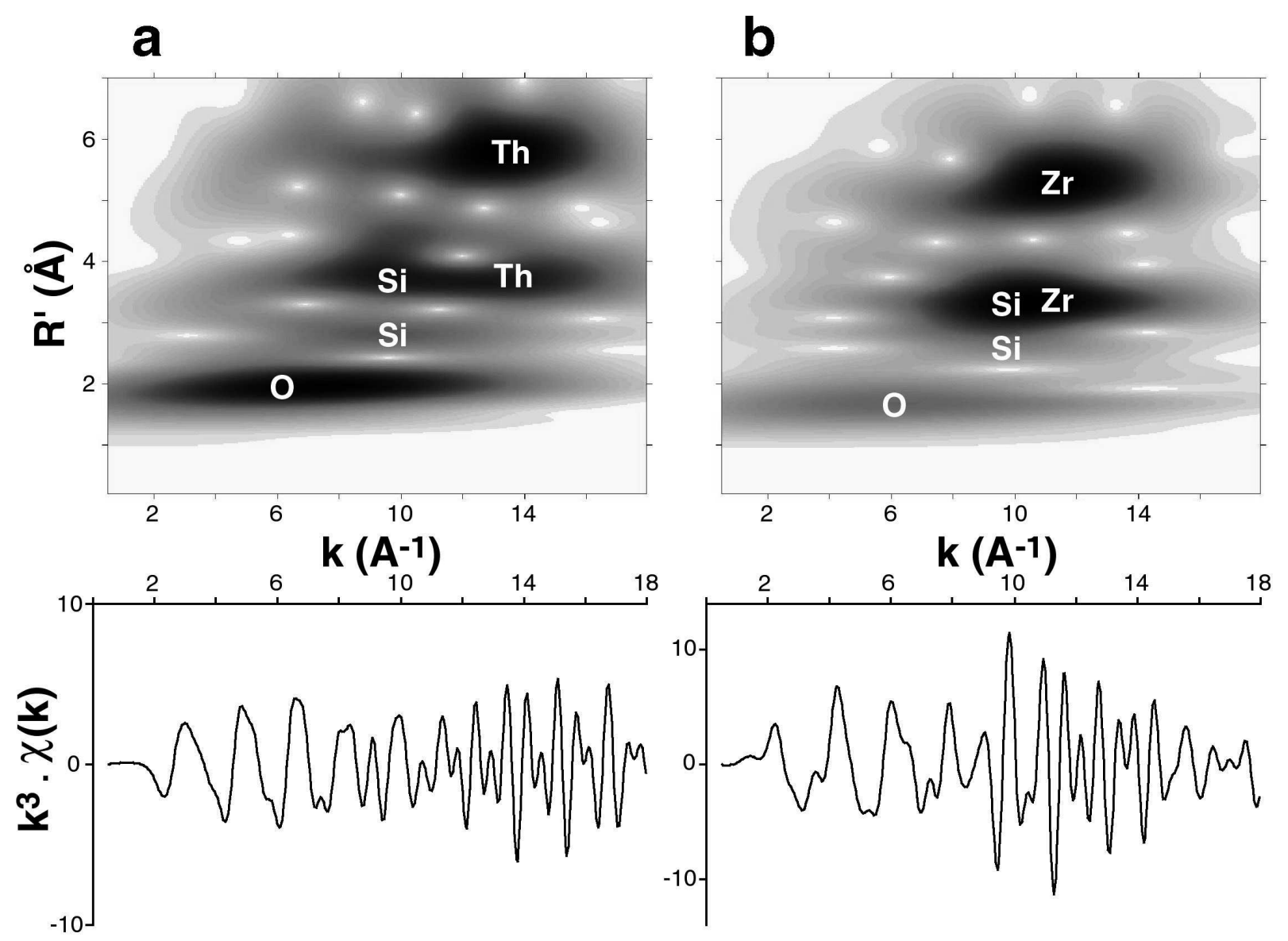


Muñoz et al. (2002) CCWT analyses of EXAFS spectra - Page 33

FIGURE 5
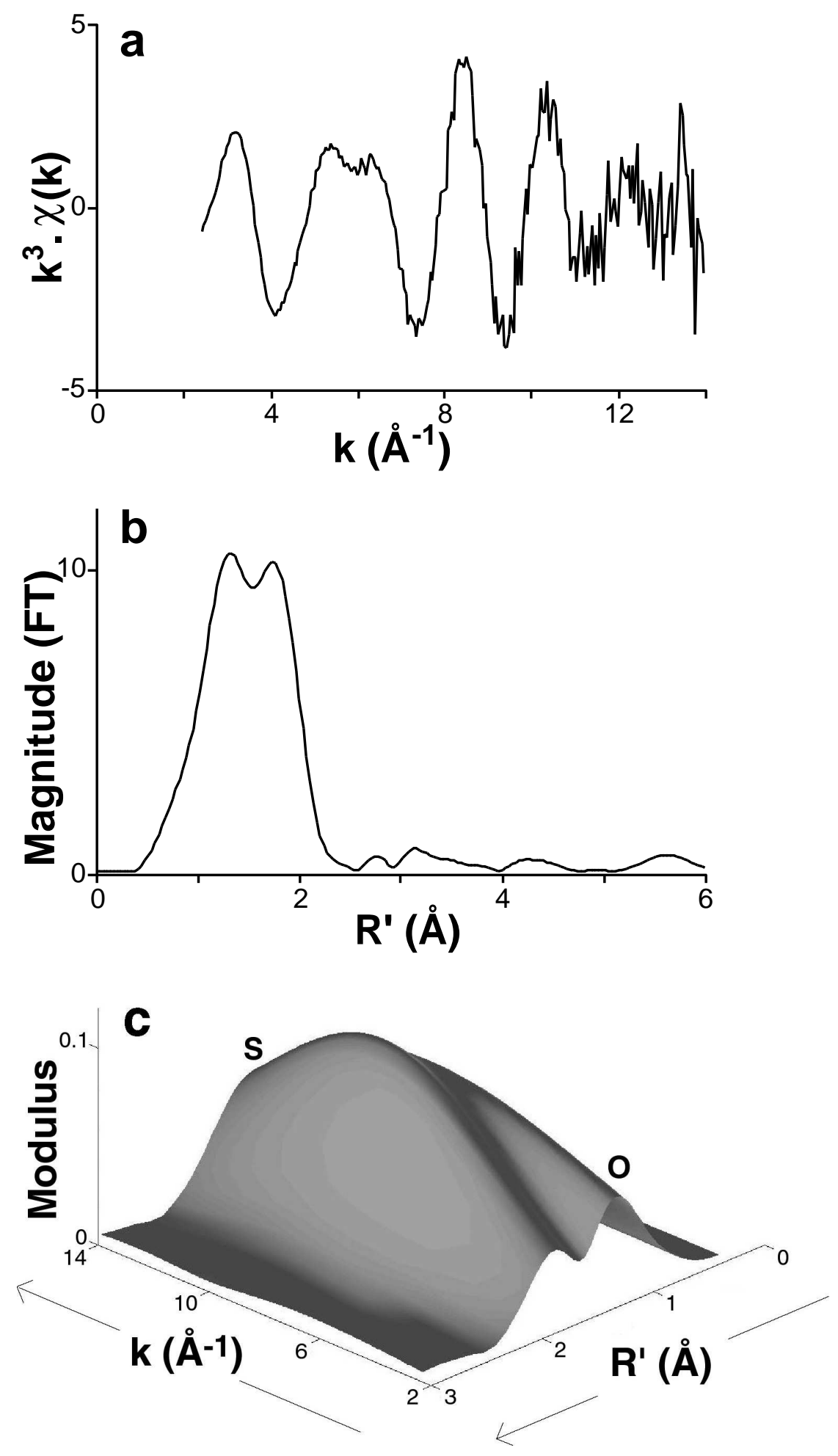
FIGURE 6

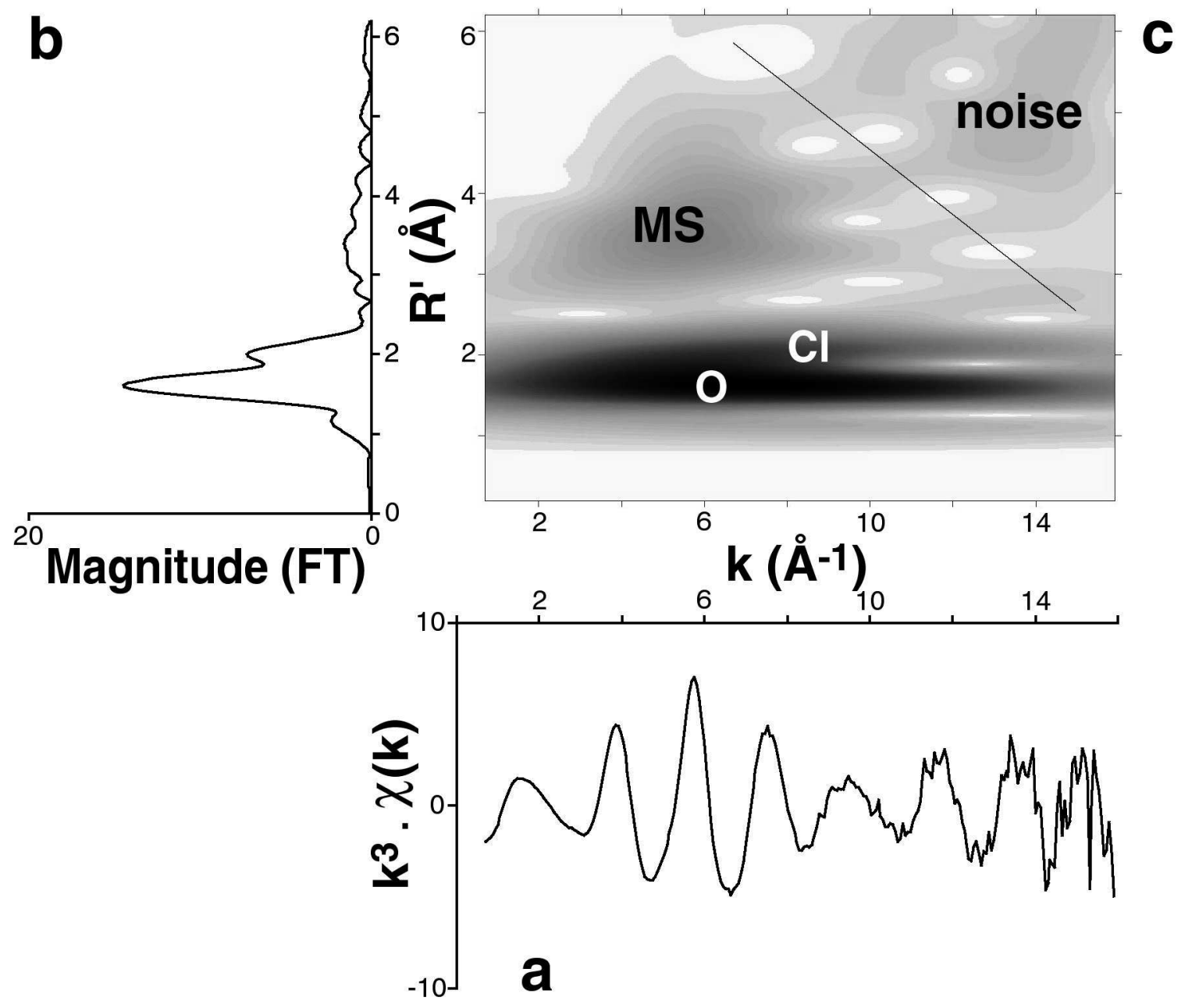

